\title{
Evaluation of a Peer Group Model of Supervision for the Allied Health Workforce in Queensland: A descriptive overview
}

\author{
Susan C. Pager \\ Metro South Health, Queensland, sue.pager@health.qld.gov.au \\ Pim Kuipers \\ Menzies Health Institute Queensland, Griffith University, Queensland, p.kuipers@griffith.edu.au \\ Karen Bell \\ Darling Downs Hospital and Health Service, Queensland, karen.bell2@health.qld.gov.au \\ Fiona Hall \\ Allied Health Professions Office of Queensland, Health Service and Clinical Excellence Division, \\ Queensland, fiona.hall@health.qld.gov.au
}

Follow this and additional works at: https://nsuworks.nova.edu/ijahsp

Part of the Health Services Research Commons

\section{Recommended Citation}

Pager SC, Kuipers P, Bell K, Hall F. Evaluation of a Peer Group Model of Supervision for the Allied Health Workforce in Queensland: A descriptive overview. The Internet Journal of Allied Health Sciences and Practice. 2018 Jan 01;16(2), Article 5.

This Manuscript is brought to you for free and open access by the College of Health Care Sciences at NSUWorks. It has been accepted for inclusion in Internet Journal of Allied Health Sciences and Practice by an authorized editor of NSUWorks. For more information, please contact nsuworks@nova.edu. 


\title{
Evaluation of a Peer Group Model of Supervision for the Allied Health Workforce in Queensland: A descriptive overview
}

\begin{abstract}
Purpose: An evaluation of a large scale implementation of a peer group model of professional supervision was conducted to inform service planning and guide policy and practice. A descriptive overview of the findings is presented. Method: Allied health staff trained in peer group supervision were surveyed about their experience of the model, its challenges and benefits. Interviews were also conducted with senior managers. Results: Analysis of 248 responses indicated that $72 \%$ of trained staff had participated in peer group supervision, and that these peer groups had continued for an average of 17.2 months. The majority of groups adhered to the guidelines presented at training, and found the model easy to implement and adaptable to a range of professions, settings and needs. Reported benefits included skill development as well as increased support and confidence. Improved relationships and team culture were also described. Management support and attendance at training were considered important to successful implementation. Conclusions: The evaluation demonstrated that a model of peer group supervision can be successfully implemented with a diverse and geographically dispersed allied health workforce. This model allowed professional supervision needs to be met in a group setting without the requirement for an expert supervisor. A number of benefits for individual clinicians and their teams were identified, and the need for further evaluation, in the context of widespread health reform is noted.
\end{abstract}

\section{Author Bio(s)}

Susan Pager BAppSc (Speech \& Hearing Science), MPH is a Senior Project Officer, Metro South Health, Brisbane, Queensland, Australia.

Pim Kuipers BA(Hons), MA, PhD is Associate Professor, Menzies Health Institute Queensland, Griffith University and Centre for Functioning \& Health Research, Metro South Health Queensland, Australia.

Karen E Bell B PHTY, MAPA is a senior physiotherapist in Darling Downs Hospital and Health Service, Queensland, Australia.

Fiona Hall DPsych, BPscy(Hons), Grad Dip Clin Hyp works with the Allied Health Professions Office of Queensland, Health Service and Clinical Excellence Division, Queensland, Australia.

\section{Acknowledgements}

The authors would like to gratefully acknowledge the support of Aly McNicoll from the New Zealand Coaching and Mentoring Centre for assistance with the training and implementation of the peer group supervision model, the Cunningham Centre for access to data and the allied health staff in Queensland who participated in this study. 


\title{
TIAHSP

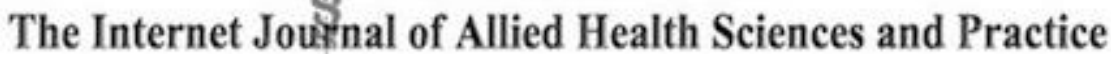

Dedicated to allied health professional practice and education

Vol. 16 No. 2 ISSN 1540-580X

\section{Evaluation of a Peer-Group Model of Supervision for the Allied Health Workforce in Queensland: A Descriptive Overview}

\author{
Susan C. Pager, BAppSc, MPH ${ }^{1}$ \\ Pim Kuipers, BA(Hons), MA, PhD² \\ Karen Bell, B PHTY, MAPA ${ }^{3}$ \\ Fiona Hall, DPsych, BPscy(Hons), Grad Dip Clin Hyp ${ }^{4}$ \\ 1. Metro South Health, Queensland \\ 2. Menzies Health Institute Queensland, Griffith University \\ 3. Darling Downs Hospital and Health Service \\ 4. Allied Health Professions Office
}

Australia

\begin{abstract}
Purpose: An evaluation of a large-scale implementation of a peer-group model of professional supervision was conducted to demonstrate service planning and guide policy and practice. A descriptive overview of the findings is presented. Method: Allied health staff trained in peer-group supervision were surveyed about the experience of the model, its challenges, and benefits. Interviews were also conducted with senior managers. Results: Analysis of 248 responses indicated that $72 \%$ of trained staff had participated in peer-group supervision and that these peer groups had continued for an average of 17.2 months. The majority of groups adhered to the guidelines presented at training and found the model easy to implement and adaptable to a range of professions, settings, and needs. Reported benefits included skill development as well as increased support and confidence. Improved relationships and team culture were also described. Management support and attendance at training were considered important to successful implementation. Conclusions: The evaluation demonstrated that a model of peer-group supervision can be successfully implemented with a diverse and geographically dispersed allied health workforce. This model was used for professional supervision needs to be met in a group setting without the requirement for an expert supervisor. A number of benefits for individual clinicians and their teams were identified and the need for further evaluation in the context of widespread health reform was noted.
\end{abstract}

\section{INTRODUCTION}

Ensuring quality professional supervision for clinicians is a priority for allied health professions. Organizationally, it will enable health care providers to meet clinical governance and quality targets. It has been associated with improved staff wellbeing, significant stress reduction, greater support and satisfaction, the maintenance of staff competence, and, importantly, best practice service delivery, and enhanced patient outcomes. ${ }^{1-4}$ Health organizations in some states of Australia have developed programs, support tools, and local policies to reinforce the provision of clinical and professional supervision. ${ }^{5}$ Queensland Health launched a professional support framework (PSF) for allied health in 2008 that was aimed at strengthening clinical governance. 6,7

A number of challenges in implementing effective supervision in the health context have been identified. $8-10$ These challenges include a lack of standardized measures, inadequate training, and limited integration with theories and models of supervision. ${ }^{11-14}$ At a practical level, they include the challenge of balancing personal boundaries, personal growth, and professional development. ${ }^{15}$ In the allied health professions, there are additional challenges for including the diversity of professional groups, the absence of a common aim, different understanding of supervision, and a preference for profession-specific approaches. ${ }^{5,16}$ 
The Queensland Health PSF was designed to enhance professional supervision for allied health professionals in the public sector. It was used to provide professional support and resources for seven different strategies. With these different strategies, the range of professional backgrounds, career stages, service contexts and geographical locations of this diverse workforce were acknowledged. ${ }^{6}$ Peer-group supervision (PGS) was included in this suite of options using a specific model under license from the New Zealand Coaching and Mentoring Centre. It was the first of the PSF strategies to have comprehensive, organization-wide training and resourcing and was offered to allied health professionals across Queensland.

Peer-group supervision has been defined as "reciprocal arrangements in which peers work together for mutual benefit where developmental feedback is emphasised and self-directed learning and evaluation is encouraged." 17 A key distinction is that PGS does not rely on a more qualified leader or identified expert. ${ }^{18}$ Reported advantages of the PGS model over individual supervision include the potential to support multidisciplinary teams while being cost and resource efficient. ${ }^{19-21}$

The particular approach of PGS implemented with allied health professionals in Queensland featured overlapping functions of learning, support, and accountability. ${ }^{18}$ The model is relatively simple, using a clear set of ground rules and seven tools for consistent implementation. This model was used to provide a common understanding, which had relevance to all allied health professions.

Based on a brief survey of allied health leaders, 8 health districts identified an interest in PGS through a train-the-trainer model. Each of these districts was asked to nominate a staff member to attend the first round of training and be part of the core team of trainers. The New Zealand Coaching and Mentoring Centre provided a two-day experiential learning workshop that was used to equip these core trainers with the necessary knowledge, skills, and competence to establish peer groups and engage in PGS. While the adoption of PGS was voluntary, Queensland Health took a coordinated statewide approach to implementation, which included establishing the core team of trainers and providing them with ongoing professional training and administrative support. Subsequent training was conducted in workshops of up to 20 participants in various facilities around Queensland. Such workshops have been advocated as a way of demystifying supervision, building competence, and enhancing implementation. ${ }^{22,23}$ The resulting peer groups were supported with the provided tools and guidelines and also assisted with the required contracting and reporting functions. In addition, a research collaborative was formed to undertake an evaluation of the quality and effectiveness of the program.

In this paper, the participation of health professionals in PGS, their experience of the groups, and the perceived benefits were described. In addition, there was documentation of some of the challenges of operationalizing PGS across a large, dispersed, diverse, and mobile workforce.

\section{METHODS}

The New Zealand Coaching and Mentoring Centre delivered a two-day train-the-trainer program to the 8 staff nominated by their districts. The program provided the experience of the training as a participant followed by small-group practice and coaching as a training facilitator. These trainers subsequently delivered the program to 613 practicing allied health professionals in the form of a one-day Power of Peer Supervision training program, which included the provision of associated resources. It was strongly recommended that staff undertake the training prior to establishing or participating in a PGS group.

Evaluation of the PGS component of the PSF was initiated 2 years after the commencement of training to allow a reasonable period of time for implementation. The evaluation was conducted by 5 senior health professionals and used mixed methods including surveys and interviews.

Ethics approval was obtained from the Gold Coast Health Service District Human Research Ethics Committee and site-specific approval from all 23 sites in Queensland. In April 2011, and after follow up in April 2012, an invitation to complete an annual online survey and consent form was emailed to all 613 staff who had completed PGS training between June 2009 and June 2011. The survey consisted of three parts. Part one was comprised of a modified version of the Clinical Supervision Evaluation Questionnaire (CSEQ), a five-point Likert-type scale that measures aspects of the "purpose," "experience," and "impact" of clinical supervision. ${ }^{24}$ Part two was comprised of questions related to group content and format and included open-ended questions to explore motivation to participate in PGS and significant changes resulting from PGS. These questions were developed based on descriptions of PGS from the literature as well as key factors included in the Manchester Clinical Supervision Scale. ${ }^{25}$ Part three was comprised of 8 demographic questions.

In 2011, semi-structured interviews were also conducted with senior managers to broaden the scope of the data and triangulate

(C) The Internet Journal of Allied Health Sciences and Practice, 2018 
methods of data collection. Interviews were conducted by telephone with a randomly selected sample of 10 allied health managers from various health districts in Queensland. They were asked about their perceptions of the PGS program and asked to identify barriers and enablers for effective PGS within their services. Recorded interviews were transcribed by an independent transcription service.

Each participant's most recent response to the annual survey was included. Descriptive analyses were applied to the resulting data set. Inductive analysis was used by the first author to identify broad themes from answers to open-ended questions in the survey and interview transcripts with the senior managers. These themes were then used by two colleagues to independently code the qualitative data. Measures of inter-coder reliability indicated strong reliability: percentage agreement (83.3\%), Fleiss' kappa (0.79), and Cohen's kappa (0.79). Combined with a measure of consistency using Krippendorf's alpha (0.79), these scores demonstrated that the coded data had a sound basis for analysis. Quotations were used to summarize themes in the words of participants, and some attributed quotations were used to provide deeper understanding of the results.

\section{RESULTS AND DISCUSSION}

A total of 152 responses were obtained for the 2011 survey (24.8\% response rate) and 149 for the 2012 survey (24.3\% response rate). Fifty-three individuals responded to both surveys, and only their most recent responses were used, resulting in a final sample for analysis of 248 responses. Demographic details of the respondents were described in terms of level of experience, profession, type of employment, type of service, and geographic location (Tables 1 and 2). The majority of respondents were women who worked in full-time permanent positions, were located in teams in metropolitan facilities, and had 11 years or more experience in their profession. Occupational therapists were the largest professional group with $49(20 \%)$ respondents, closely followed by social workers (19\%), physiotherapists $(13 \%)$, and psychologists (10\%). Some large professional groups were not represented (pharmacists, $0 \%$ ) or had limited representation (radiographers, $1 \%$ ), which may suggest the PGS model is less suitable for or of less interest to these professions.

Table 1. Demographic Features of Survey Respondents

\begin{tabular}{|c|c|c|}
\hline 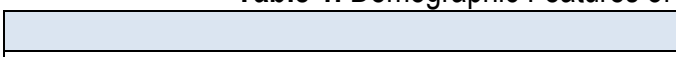 & $n$ & $\%$ \\
\hline \multicolumn{3}{|l|}{ Sole Practitioner } \\
\hline Yes & 49 & 20 \\
\hline No & 180 & 73 \\
\hline \multicolumn{3}{|l|}{ Location } \\
\hline Metropolitan & 198 & 80 \\
\hline Regional or remote & 31 & 13 \\
\hline \multicolumn{3}{|l|}{ Gender } \\
\hline Female & 198 & 80 \\
\hline Male & 31 & 13 \\
\hline \multicolumn{3}{|l|}{ New Graduate } \\
\hline Yes & 8 & 3 \\
\hline No & 221 & 90 \\
\hline \multicolumn{3}{|l|}{ Level of Position } \\
\hline HP3 & 40 & 16 \\
\hline HP4 & 116 & 47 \\
\hline HP5 & 59 & 24 \\
\hline HP6 or above (includes $1 \times$ HP7) & 9 & 4 \\
\hline Other (TO2, 004, NO, student) & 5 & 2 \\
\hline \multicolumn{3}{|l|}{ Years of Experience in Current Profession } \\
\hline $0-2$ years & 12 & 5 \\
\hline $3-5$ years & 34 & 14 \\
\hline
\end{tabular}

(c) The Internet Journal of Allied Health Sciences and Practice, 2018 


\begin{tabular}{|l|c|c|}
\hline 6-10 years & 55 & 22 \\
\hline 11 or more years & 128 & 52 \\
\hline \multicolumn{2}{|l|}{} \\
\hline \begin{tabular}{l|l|} 
Current Employment Status \\
Full-time permanent
\end{tabular} & 155 & 63 \\
\hline Full-time casual or contract & 17 & 7 \\
\hline Part-time permanent & 48 & 19 \\
\hline Part-time casual or contract & 5 & 2 \\
\hline Other (holds more than 1 position) & 4 & 2 \\
\hline
\end{tabular}

Note: $\mathrm{n}=229$. Missing $=19$.

Table 2. Profession of Survey Respondents

\begin{tabular}{|l|c|c|}
\hline \multicolumn{1}{|c|}{ Professions } & $\boldsymbol{n}$ & $\%$ \\
\hline Therapy assistant & 1 & $<1$ \\
\hline Audiologist & 2 & $<1$ \\
\hline Counsellor or family therapist & 0 & 0 \\
\hline Clinical measurement & 1 & $<1$ \\
\hline Dental/oral therapist & 2 & $<1$ \\
\hline Dietitian & 23 & 9 \\
\hline Exercise physiologist & 1 & $<1$ \\
\hline Health promotion officer & 0 & 0 \\
\hline Health worker & 1 & $<1$ \\
\hline Nutritionist & 8 & 3 \\
\hline Occupational therapist & 49 & 20 \\
\hline Pharmacist & 0 & 0 \\
\hline Physiotherapist & 33 & 13 \\
\hline Podiatrist & 5 & 2 \\
\hline Prosthetist/orthotist & 0 & 0 \\
\hline Psychologist & 26 & 10 \\
\hline Radiation therapist & 0 & 0 \\
\hline Radiographer & 3 & 1 \\
\hline Registered nurse & 1 & $<1$ \\
\hline Social worker & 46 & 19 \\
\hline Speech pathologist & 21 & 8 \\
\hline Other (team leader, library technician) & 7 & 3 \\
\hline
\end{tabular}

Note: $n=230$. Missing $=18$.

\section{Participation in Peer-Group Supervision}

Seventy-two percent of staff who responded to the surveys reported that they participated in a peer group following training. Acknowledging a positive response bias, this rate is still considerably higher than described in studies with genetic counselors $(34.8 \%)$ and nurses (38\%). ${ }^{26,27}$ These findings indicate that the professional support framework and policy imperative in Queensland may have led to a relatively high level of interest.

Respondents who had considerable engagement in a peer group identified personal benefits, such as enjoyment, individual support, and opportunity for reflection.

"It is a valuable way to learn and grow in a peer environment where there is recognition that each participant is equal." Metropolitan HP5 Occupational Therapist 
There were also identified benefits to clients and to the organization.

"It was beneficial to personal professional practice, patient care, and job satisfaction. Also helps with multidisciplinary awareness and networking." Regional HP4 Speech Pathologist

While less than $10 \%$ of trainees indicated they had no interest in being in a peer group, it was "real-world" implementation and included negative responses. For some, PGS was the only available option and "better than nothing," and others said that they were directed to attend by management as it was a "requirement placed on staff," or they were obliged to participate to maintain professional registration. Comments from those with limited interest often related to the perception that the model did not suit their current needs or was difficult to arrange.

"It is hard to find time. And it is important to find the right people to be in your group, otherwise it is a waste of time." Metropolitan HP4 Occupational Therapist

There were 70 respondents (28\%) who indicated they had previously been in a peer group but were no longer active. Some of the respondents reported they had changed jobs or location (33\%), turnover of staff had disrupted the group, or their peer group had "just petered out'" (29\%) often due to time issues and competing demands. Less frequently, they reported that the group made a decision to discontinue (14\%), or an individual member chose to stop attending a group (6\%). Some respondents provided specific reasons for group cessation, and these reasons included time pressures, staff changes, and lack of adherence to the model.

At the time of the survey, PGS groups had been running for an average of 17.2 months (range $=1-77$ months). Some respondents reported they had participated in several groups, forming new groups, or joining existing groups as they changed location or as groups ceased.

"[I] have been in 3 different groups now. [I] have helped form them. [I] still participate regularly in 2 PGS groups." Metropolitan HP5 Psychologist

Group composition did not appear to be important for group success. Different types of groups were reported as successful: both single discipline and multi-professional groups, co-located and geographically dispersed groups, those with similar and mixed levels of experience, and groups from closely aligned and dissimilar services. Analysis of the interview feedback indicated that success of the PGS group model was more related to support from managers than many participant factors. Participants noted that "ongoing support by a facilitator to get going" and to follow the procedure was important and that "working with the training and resources provided has really helped the group run effectively."

\section{The Experience of Peer-Group Supervision}

The majority of participants indicated they adhered to the guidelines presented in the training, including location, using the tools as provided, always having a facilitator, and providing for several group members to present issues at each session. Participants reported that their peer groups were usually conducted in a quiet, private space within the workplace (74\%). Groups were rarely held outside of the workplace ( $9 \%)$, and only $4 \%$ utilized videoconferencing or teleconferencing facilities. Peer groups generally lasted between 1 and 2 hours (65\%), although some were less than 1 hour (17\%) or more than 2 hours (5\%). Peer groups were mostly held on a monthly basis $(55 \%)$.

The structure of the model was valued by participants.

"Our group is still new and developing; however, I have found it is very beneficial. I have found that it is most effective when structure and rules are followed." Metropolitan HP4 Social Worker

Peer groups most often had 4 to 6 members (70\%) as recommended by the PGS guidelines. Composition of groups varied across single-profession groups (57\%): groups comprised of two professions (28\%) and occasionally groups with more than 4 different professions (5\%).

\section{The Challenges of Implementing Peer-Group Supervision}

The implementation of PGS on such a large scale was associated with a number of challenges. Analysis of the survey responses identified a number of enabling and limiting factors considered to have affected the overall success of the program. Following the training, $71 \%$ of respondents reported being very or moderately confident to be part of a peer group, which was attributed to the training via an experiential face-to-face workshop and the strength of the model. Staff members who did not feel confident

(c) The Internet Journal of Allied Health Sciences and Practice, 2018 
with PGS were often critical of the model or had not actually tried a peer group. Other reasons for low confidence included feeling unhappy with how their peer group had been formed and lack of adherence to the processes: "just more chatting, never following the structural PGS protocol."

Interestingly, attempts by managers (rather than peers) to organize peer groups and influence group membership were often viewed negatively by participants and were linked with problems.

"The group wasn't joined voluntarily, and the group members were staff members that did not necessarily work well together. This led to some hostility and mistrust within the group. This manifested as a lack of willingness for any member to take on the responsibility of organising the dates and locations for the meeting." Metropolitan HP4 Audiologist

However, support from managers in the form of releasing staff from clinical duties to attend, providing appropriate meeting locations, and allowing choice in the membership of groups was regarded positively. After the analysis of interview data, we concluded that the support of managers, team leaders, and the organization in promoting the model and reducing barriers to implementation was a critical success factor. These findings are consistent with previous observations of clinical supervision models. ${ }^{23}$

Approximately one-third (31\%) of respondents reported they had experienced a problem in their group at some time. These problems usually pertained to difficulty with the delivery or content of the model and included interruptions, time constraints, not adhering to the format (which was considered tedious at times), and lack of preparation for sessions. Difficulty with group logistics and attendance was also a negative effect (members being dispersed across geographical locations, frequent changes of membership, restricted availability of part-time staff, and competition from other workload demands). Sometimes peer groups did not meet the perceived professional support needs of members. Often this perception was observed when the groups were not formed voluntarily or dwelt on the concerns of less experienced group members at the expense of the more experienced. Some participants noted that groups had been able to resolve problems through modification of membership, meeting times, and rules; however, there were also instances in which the groups were unhelpful. As noted in the PGS materials, "There is potential for peer review groups to be harmful if there is inadequate attention to process within the group."14,18,23

\section{The Benefits of Peer-Group Supervision}

Most respondents reported experiencing professional benefits from participating in PGS. These included skill acquisition (both clinical and supervisory skills), professional development, and meeting the requirements of professional registration bodies. Managers and some regional respondents considered peer groups beneficial because they lacked suitably skilled and available professionals to provide one-on-one supervision.

The flexibility of the model was reported as a benefit, which enabled participation across professional boundaries.

"I think peer supervision will become increasingly important as the number of interprofessional teams and the degree of interprofessional practice rises." Metropolitan HP5 Physiotherapist

When respondents were asked why they participated in PGS, three key themes emerged.

- First, a peer group was considered to provide support in a safe, objective, and shared way for a range of issues, particularly in times of stress. This support was perceived as leading to improved confidence and job satisfaction.

- Second, the PGS model was believed to be an efficient, beneficial, structured, and balanced approach that can be targeted to specific group needs, supplementing other professional support, or filling the gap if no other support were available. The model had a relatively simple set of skills and tools and encouraged flexibility in implementation, which resulted in a range of successful groups across different locations, levels of experience, and mix of professionals involved.

- Third, peer group participation was used to develop skills, improve practice knowledge, meet professional development requirements of professional bodies, learn about other disciplines, and ultimately to improve patient care.

These benefits were consistent with those resulting from other forms of professional support, such as expert-led group supervision in nursing care and process-oriented group supervision for nursing students. ${ }^{9,29,30}$

(c) The Internet Journal of Allied Health Sciences and Practice, 2018 
"I am much more confident in exploring difficult issues with my PGS colleagues. I also find myself much more open to alternative possibilities that I may otherwise not have explored or not felt achievable." Metropolitan HP 7 Physiotherapist

Those with experience of PGS were also asked to identify the most significant change for them as a result of their participation. A number of themes emerged, including improved sense of team collaboration, relationships, and culture within the team. Other significant changes related to increased role clarity, participation, sharing, cohesion, listening, trust, appreciation, and accountability to other group members. Learning new skills, constructive problem solving, and dealing with conflict were also noted as positives. Importantly, respondents also identified significant changes to their way of thinking. They reported taking time to reflect on practice, appreciating broader perspectives, and gaining insights into their own strengths and weaknesses. Positive emotional changes included feeling more confident, validated, empowered, and satisfied with their work as well as reduced stress levels. A small number of respondents (8\%) reported experiencing no significant change as a result of their participation in PGS, or they had not yet had enough experience.

\section{CONCLUSIONS}

This descriptive overview of an evaluation study indicated that a model of PGS can be successfully implemented for a diverse and geographically dispersed allied health workforce. A number of key issues can be interpreted. First, the embedding of PGS in a broader, comprehensive framework of "professional support," including policy development, created an organizational imperative for professional support while at the same time, professional and individual preferences were being accommodated. Multi-professional and single-profession groups were both considered to be effective and easily accommodated by the PGS model. Second, the large-scale implementation over the initial two-year period facilitated a critical mass of trained clinicians who then confidently and positively influenced the supervision culture within their workplaces. With this model, the professional support needs of a large workforce were allowed to be met in a group setting without the need for expert supervisors. We suggest there are considerable benefits in the PGS model for individual clinicians and for their teams. There is a need for further research to explore the effects of PGS on the quality of care and patient outcomes.

With this descriptive overview of the evaluation and associated studies, there still remains a call for studies to be larger in scale and to incorporate valid and reliable instruments to measure change. ${ }^{25,30,31}$ By providing a comprehensive description and evaluation of a large scale PGS program, the findings of this study have contributed significantly to the body of knowledge. Further evaluation of the model's sustainability in the context of widespread health reform is recommended.

\section{REFERENCES:}

1. Francke AL, de Graaff FM. The effects of group supervision of nurses: a systematic literature review. Int J Nurs Stud.2012;49(9):1165-79. [PMID: 22177568]

2. Blomberg $K$, Isaksson $A K$, Allvin $R$, Bisholt $B$,Ewertsson $M$, et al. Work stress among newly graduated nurses in relation to workplace and clinical group supervision. J Nurs Manag. 2016;24(1):80-7. [PMID:25421164]

3. Kilminster SM, Jolly BC. Effective supervision in clinical practice settings: a literature review. Med Educ. 2000;34(10):827-40. [PMID: 11012933]

4. Cleary M, Freeman A. The cultural realities of clinical supervision in an acute inpatient mental health setting. Issues Ment Health Nurs. 2005;26(5):489-505. [PMID: 16020064]

5. Fitzpatrick S, Smith M, Wilding C. Quality allied health clinical supervision policy in Australia: a literature review. Aust Health Rev. 2012;36(4):461-5. [PMID: 23116979]

6. Hall F, Bell K. Professional support framework: improving access to professional support for professionals. Aust Health Rev. 2013;37(5):560-5. [PMID 23680624]

7. Bell KE, Hall F, Pager S, Kuipers P, Farry H. Developing allied health professional support policy in Queensland: a case study. Hum Resour Health. 2014 Oct 8;12:57. [PMID: 25296763]

8. Barnes MW, E Winstanley, J Reed, R. Clinical supervision and continuing professional development for midwives in Queensland, Australia: Findings from an online survey. Focus on Health Professional Education: A Multi-professional Journal. 2013;14(2):1-11.

9. Wolfe M, Lavery J, Darra S. Exploring the value of group supervision in midwifery. Part 2: Evaluation of data from Wales. $\mathrm{Br} \mathrm{J}$ Midwifery. 2016;24(4):288-97.

10. White E, Roche M. A selective review of mental health nursing in New South Wales, Australia, in relation to clinical supervision. Int J Ment Health Nurs. 2006;15(3):209-19. [PMID: 16916415]

11. Bogo M, McKnight K. Clinical supervision in social work: a review of the research literature. Clin Superv. 2005;24(1/2):49-67.

12. Yegdich T, Cushing A. An historical perspective on clinical supervision in nursing. Aust N Z J Ment Health Nurs. 1998;7(1):3-

(C) The Internet Journal of Allied Health Sciences and Practice, 2018 
24. [PMID: 9708063]

13. Buus $\mathrm{N}$, Gonge $\mathrm{H}$. Empirical studies of clinical supervision in psychiatric nursing: A systematic literature review and methodological critique. Int J Ment Health Nurs. 2009;18(4):250-64. [PMID: 19594645]

14. Sweeney G, Webley P, Treacher A. Supervision in occupational therapy, part 2: The supervisee's dilemma. Br J Occup Ther. 2001;64(8):380-6.

15. Morcom C, Hughes R. How can clinical supervision become a real vision for the future? J Psychiatr Ment Health Nurs.1996;3(2):117-24. [PMID 8716803]

16. Fitzpatrick S, Smith M, Wilding $C$. Clinical supervision in allied health in Australia: A model of allied health clinical supervision based on practitioner experience. IJAHSP. 2015;13(4).

17. Benshoff JM. Peer Consultation as a Form of Supervision. Ann Arbor, MI: ERIC/CASS. 1992.

18. McNicoll A. Peer Supervision - No-one knows as much as all of us. 2008. Available at http://www.coachingmentoring.co.nz/resources/articles/134-peer-supervision-no-one-knows-as-much-as-all-of-us.Accessed October 24, 2017.

19. Thomas B, Reid J. Multidisciplinary clinical supervision. Br J Nurs. 1995;4(15):883-5.

20. White $\mathrm{E}$, Winstanley J. Cost and resource implications of clinical supervision in nursing: an Australian perspective. J Nurs Manag. 2006;14(8):628-36. [PMID: 17054736]

21. Lavery J, Wolfe M, Darra S. Exploring the value of group supervision in midwifery: Part 1. Br JMidwifery.2016;24(3):196202.

22. Taylor M, Harrison CA. Introducing clinical supervision across Western Australian public mental health services. Int J Ment Health Nurs. 2010;19(4):287-93. [PMID: 20618529]

23. White $\mathrm{E}$, Winstanley J. Implementation of Clinical Supervision: educational preparation and subsequent diary accounts of the practicalities involved, from an Australian mental health [corrected] nursing innovation. J Psychiatr Ment Health Nurs. 2009;16(10):895-903. [PMID: 19930363]

24. Horton S, de Lourdes Drachler M, Fuller A, de Carvalho Leite JC. Development and preliminary validation of a measure for assessing staff perspectives on the quality of clinical group supervision. Int J Lang Commun Disord.2008;43(2):126-134. [PMID: 17852531]

25. Winstanley J, White E. The MCSS-26: revision of the Manchester Clinical Supervision Scale using the Rasch Measurement Model. J Nurs Meas. 2011;19(3):160-78. [PMID: 22372092]

26. Zahm KW, Veach PM, LeRoy BS. An investigation of genetic counselor experiences in peer group supervision. J Genet Couns. 2008;17(3):220-33. [PMID: 17701451]

27. Davey B, Desousa C, Robinson S, Murrells T. The policy-practice divide: Who has clinical supervision in nursing? J Res Nurs. 2006;11(3):237-48.

28. Scanlon C, Weir WS. Learning from practice? Mental health nurses' perceptions and experiences of clinical supervision. $J$ Adv Nurs. 1997;26(2):295-303. [PMID: 9292363]

29. Arvidsson B, Lofgren H, Fridlund B. Psychiatric nurses' conceptions of how a group supervision programme in nursing care influences their professional competence: a 4-year follow-up study. J Nurs Manag. 2001;9(3):161-71. [PMID: 11879463]

30. Arvidsson B, Baigi A, Skarsater I. Changes in the effects of process-oriented group supervision as reported by female and male nursing students: a prospective longitudinal study. Scand J Caring Sci. 2008;22(3):437-44. [PMID:18840227]

31. Kuipers $\mathrm{P}$, Pager $\mathrm{S}$, Bell K, Fiona. $\mathrm{H}$, Kendall $\mathrm{M}$. Do structured arrangements for multidisciplinary peer group supervision make a difference for allied health professional outcomes? J Multidiscip Healthc. 2013;6:391-7. [PMID: 24143110] 